\title{
Amyloid $\beta$ deposition and glucose metabolism on the long-term progression of preclinical Alzheimer's disease
}

Future Science $\mathrm{OA}$

\author{
Shizuo Hatashita*,1 \& Daichi Wakebe ${ }^{2}$ \\ ${ }^{1}$ Department of Neurology, Shonan-Atsugi Hospital, Atsugi 243-0033, Japan \\ ${ }^{2}$ Department of Radiology, Shonan-Atsugi Hospital, Atsugi 243-0033, Japan \\ *Author for correspondence: Tel.: +81 46223 3636; Fax: +81 46223 3630; shizu@olive.ocn.ne.jp
}

\begin{abstract}
Aim: Longitudinal changes in beta amyloid $(A \beta)$ deposition and glucose metabolism over a long-term progression of preclinical Alzheimer's disease (AD) were evaluated. Methods: 22 preclinical AD subjects with amyloid-positive scans underwent $\left[{ }^{11} \mathrm{C}\right]$-labeled Pittsburgh Compound-B (PIB) positron emission tomography (PET) and $\left[{ }^{18} \mathrm{~F}\right]$-fluorodeoxyglucose (FDG) PET imaging over $6.0 \pm 1.8$ years. A quantitative analysis of $\left[{ }^{11} \mathrm{C}\right]$-PIB and $\left[{ }^{18} \mathrm{~F}\right]-\mathrm{FDG}$ was used with a standardized uptake value ratio (SUVR) in the same regions. Results: In preclinical AD subjects, the cortical PIB SUVR was higher at baseline and increased at followup. 12 of the preclinical AD subjects progressed to mild cognitive impairment, six of whom had reduced glucose metabolism. The annual change in PIB SUVR was not related to that in FDG SUVR. Conclusion: Increases in $A \beta$ deposition lead to the progression to mild cognitive impairment, but decreases in glucose metabolism do not contribute to progression.
\end{abstract}

Lay abstract: Longitudinal changes in $A \beta$ deposition and glucose metabolism were clarified over a longterm progression of preclinical AD using $\left[{ }^{11} \mathrm{C}\right]-\mathrm{PIB}$ PET and $\left[{ }^{18} \mathrm{~F}\right]-$ FDG PET imaging. $73 \%$ of the preclinical AD subjects progressed to mild cognitive impairment during 6 years of a long follow-up period. The increases in $A \beta$ deposition lead to the progression to mild cognitive impairment but decreases in glucose metabolism do not contribute to progression.

First draft submitted: 27 June 2018; Accepted for publication: 11 October 2018; Published online: 19 February 2019

Keywords: Alzheimer's disease $\bullet$ amyloid $\beta$ deposition $\bullet$ glucose metabolism $\bullet$ preclinical AD

The National Institute on Aging Alzheimer's Association (NIA-AA) workgroup has proposed diagnostic criteria for the spectrum of Alzheimer's disease (AD) supported by distinctive and reliable biomarkers of AD [1]. In cognitively normal $(\mathrm{CN})$ individuals who have evidence of beta amyloid $(\mathrm{A} \beta)$ accumulation, the operational framework for the preclinical phase of $\mathrm{AD}$ has been demonstrated in an asymptomatic or latent form of the disease as preclinical $\mathrm{AD}$ [2]. The preclinical stage of $\mathrm{AD}$ has a time lag of a decade or more between the beginning of the $\mathrm{AD}$ pathophysiological process and the onset of evident clinical impairment. The long preclinical phase of AD allows potential intervention with disease-modifying therapy that treats the early pathological process to prevent subsequent neurodegeneration and eventual cognitive decline. It is important for the pathophysiological process in the preclinical stage of $\mathrm{AD}$ to be evaluated with molecular positron emission tomography (PET) imaging of $\mathrm{A} \beta$ accumulation and neurodegeneration.

Amyloid PET imaging with a tracer of $\left[{ }^{11} \mathrm{C}\right]$-labeled Pittsburgh Compound-B (PIB), which has high affinity for fibrillar $A \beta$, is a reliable biomarker of $A \beta$ accumulation [3]. We previously reported that a diagnostic framework with $\mathrm{A} \beta$ deposition by $\left[{ }^{11} \mathrm{C}\right]$-PIB PET at different clinical stages of $\mathrm{AD}$ enables an earlier and more specific $\mathrm{AD}$ diagnostic process [4]. Furthermore, in the patients with mild cognitive impairment (MCI) due to AD defined by [11C]-PIB PET, our recent work has demonstrated that a substantial increase in brain $A \beta$ deposition has an effect on cognitive decline and disease progression [5]. In addition, if $\mathrm{CN}$ individuals are identified as preclinical $\mathrm{AD}$ with a positive $\mathrm{A} \beta \mathrm{PET}$ scan, they may be at risk for progression to $\mathrm{MCI}$ or $\mathrm{AD}$ dementia over a long term. 
Fluorodeoxyglucose (FDG) PET imaging for cerebral glucose metabolism, which is one of the biomarkers for neurodegeneration, provides evidence of cognitive function and progression along the spectrum of AD. FDG PET has revealed a decrease in cerebral glucose metabolism with a characteristic regional pattern of temporoparietal hypometabolism in patients with pathologically confirmed AD [1]. Notable changes in FDG PET scans occur only after subjects become symptomatic. In contrast, in presymptomatic subjects, a relationship between $\left[{ }^{11} \mathrm{C}\right]-\mathrm{PIB}$ and $\left[{ }^{18} \mathrm{~F}\right]-\mathrm{FDG}$ on PET imaging has been previously reported [6]. We have demonstrated that most patients with MCI due to $A D$ based on $A \beta$ and FDG PET biomarkers have a progression to $A D$, even over the short term [7]. Studies have also reported that $\mathrm{CN}$ individuals with markers for both amyloid deposition and neurodegeneration are more likely to develop cognitive impairments during short follow-up periods $[8,9]$. However, a longitudinal study of A $\beta$ deposition and cerebral glucose metabolism in the subjects with preclinical AD defined by amyloid PET has not been conducted over a long follow-up period.

We defined preclinical AD using amyloid PET imaging for an $\mathrm{A} \beta$ biomarker in $\mathrm{CN}$ individuals and evaluated the longitudinal change in $A \beta$ deposition and glucose metabolism over the long progression of preclinical $A D$ using PIB PET and FGD PET imaging. In addition, we sought to determine whether $A \beta$ deposition and glucose metabolism are associated with the progression to $\mathrm{MCI}$ or $\mathrm{AD}$ dementia in the preclinical phase of $\mathrm{AD}$.

\section{Materials \& methods}

Subjects

$\mathrm{CN}$ subjects between 60 and 89 years of age were recruited from community-dwelling individuals. The subjects underwent cognitive testing and $\left[{ }^{11} \mathrm{C}\right]-\mathrm{PIB}$ PET. The normal cognitive status of $\mathrm{CN}$ subjects was defined by a Mini-Mental State Examination (MMSE) score of 28 or more and a clinical dementia rating (CDR) score of 0. Of these subjects, $22 \mathrm{CN}$ subjects who had an amyloid-positive scan were defined as preclinical $\mathrm{AD}$ and subsequently included in the long-term follow-up study. Ten $\mathrm{CN}$ subjects who had an amyloid-negative scan were also included as the negative $\mathrm{CN}$ group. All $\mathrm{CN}$ subjects were clinically assessed every 6 months and underwent $\left[{ }^{11} \mathrm{C}\right]-\mathrm{PIB}$ PET and $\left[{ }^{18} \mathrm{~F}\right]$-FDG PET imaging at baseline and at least once during the $6.0 \pm 1.8$ years of follow-up (range 3.1-8.8 years). The apolipoprotein E (APOE) genotype was determined. Participants were excluded if they had a neurodegenerative condition, stroke, psychiatric illness, traumatic brain injury or any other medical condition. Each subject provided written informed consent for participation. The study was approved by the Ethics Committee of the Mirai Iryo Research Center Inc. (Tokyo, Japan).

MCI was diagnosed at each visit if the CN subjects fulfilled the Core Clinical Criteria for MCI proposed by the NIA-AA workgroup [10]. The MMSE score was 24 or more, and the global CDR score was at least 0.5 in the memory domain. Delayed recall of a paragraph from the revised Wechsler memory scale (WMS-R) Logical Memory II (maximum score 25) was used as the measure of the episodic memory. AD dementia was diagnosed based on the criteria of the NIA-AA. AD patients had an MMSE score of 23 or less, a global CDR score of 0.5 or 1 and impaired activities of daily living.

\section{PET imaging}

All PET scans were performed with a Siemens ECAT ACCEL scanner on the same day as cognitive testing. All imaging data were reconstructed into a $128 \times 128$ matrix with an iterative reconstruction algorithm, using a Gaussian filter with 3.5-mm full-width at half-maximum. The subject's head was immobilized to minimize motion during the scan. Amyloid PET imaging was performed with the radiotracer $\left[{ }^{11} \mathrm{C}\right]-\mathrm{PIB}[4]$. The $\left[{ }^{11} \mathrm{C}\right]-\mathrm{PIB}$ was injected with a mean dose of $550.0 \pm 10 \%$ MBq. Dynamic PET scanning was performed for 60 min using a predetermined protocol. Sixty minutes after the completion of the $\left[{ }^{11} \mathrm{C}\right]-\mathrm{PIB}$ PET scan, subjects were injected with $250.0 \pm 10 \% \mathrm{MBq}$ of $\left[{ }^{18} \mathrm{~F}\right]-\mathrm{FDG}$ and remained in a dark room. Fifteen-minute static FDG PET scans were acquired after a 45 -min uptake period.

\section{Image analysis}

A region of interest (ROI) analysis was performed on each individual PET image. All subjects underwent T1weighted MRI (1.5 T) for coregistration with the PET images. MRI-based correction of the PET data was carried out using PMOD software (PMOD Technologies Ltd, Adliswil, Switzerland). The ROIs were manually drawn on the coregistered MRI of each subject for 20 bilateral cortical regions including the lateral temporal cortex (LTC), medial temporal cortex (MTC), frontal cortex (FC), occipital cortex (OC), parietal cortex (PC), sensory motor cortex (SMC), anterior cingulate gyrus (ACG), posterior cingulate gyrus (PCG), precuneus cortex (Pre) and 
cerebellar cortex. The cerebellar gray matter was used as a reference region. The ROIs of the follow-up PET images were coregistered with the initial PET images, and the same ROIs were applied to both the baseline and follow-up images.

The retention of $\left[{ }^{11} \mathrm{C}\right]-\mathrm{PIB}$ was calculated as the regional-to-cerebellum standardized uptake value ratio (SUVR) for 35-60 min. The regional PIB SUVR in each cortical region and the global cortical PIB SUVR for the mean of the regional SUVR over 18 cortical regions, including LTC, MTC, FC, OC, PC, SMC, ACG, PCG and Pre, were defined. The same coregistration method was applied to the quantification of $\left[{ }^{18} \mathrm{~F}\right]$-FDG. A standardized uptake value of the same regions was normalized to the cerebellar cortex as a reference. Glucose metabolism was referred to as the SUVR, and the global and regional cortical FDG SUVRs were defined. The decrease in glucose metabolism was less than the $\left[{ }^{18} \mathrm{~F}\right]$-FDG SUVR of 0.99 in the cortical regions in our clinical setting, as previously described [7].

The amyloid-positive scan had an increased $\left[{ }^{11} \mathrm{C}\right]$-PIB SUVR of 1.39 or more in any of the cortical regions at baseline and was classified as a typical or focal positive scan in our clinical setting. The amyloid-negative scan had no $\left[{ }^{11} \mathrm{C}\right]-\mathrm{PIB}$ uptake in any cortical region. The cut-point value was based on the bimodal distribution of $\left[{ }^{11} \mathrm{C}\right]$-PIB uptake in $56 \mathrm{CN}$ controls and $32 \mathrm{AD}$ patients, and it discriminated $\mathrm{AD}$ patients from healthy controls with a sensitivity of $97.2 \%$ and a specificity of $85.3 \%$, as previously described [11].

\section{Data management}

The subjects underwent clinical assessments and $\left[{ }^{11} \mathrm{C}\right]$-PIB PET and $\left[{ }^{18} \mathrm{~F}\right]$-FDG PET imaging at three to six time points approximately 12 months apart during the follow-up period. Annual changes in the $\left[{ }^{11} \mathrm{C}\right]-\mathrm{PIB}$ and $\left[{ }^{18} \mathrm{~F}\right]$-FDG SUVRs of each cortical region were calculated for each subject at the final follow-up visit using the following equation: annual change $=([$ SUVR at last follow-up - SUVR at baseline $] /$ follow-up period [year] $)$.

\section{Statistical analysis}

Data were analyzed with Statcel 3 software (OMS, Inc.,Tokyo, Japan). Paired $t$ tests were used to study changes between baseline and follow-up data. Group differences were evaluated with Bonferroni post hoc tests. Pearson's correlation analyses were conducted for PIB SUVRs, FDG SUVRs, baseline age, MMSE scores, CDR sum of boxes scores and WMS-R recall scores. Categorical variables were examined with Fisher's exact test. The results were considered significant at $\mathrm{p}<0.05$. Data are presented as the mean $\pm \mathrm{SD}$.

\section{Results}

\section{Clinical data \& cognitive function}

The demographic characteristics of preclinical $\mathrm{AD}$ and amyloid-negative $\mathrm{CN}$ subjects at baseline and at follow-up are shown in Table 1. All preclinical AD subjects had a mean age of $71.0 \pm 5.9$ (range, 62-87) and had no cognitive impairment on MMSE, global CDR or WMS-R Logical Memory II Immediate and Delayed Recall. The proportion of APOE $\varepsilon 4$ carriers was greater in preclinical AD subjects ( $41 \%)$ than in $\mathrm{CN}$ subjects $(10 \%)$.

$12(55 \%)$ of the 22 subjects with preclinical $\mathrm{AD}$ had a progression to MCI during the mean follow-up of $6.0 \pm 1.8$ years (converters), whereas the other ten subjects remained clinically stable (stable). The pattern of the consecutive progression rate of preclinical AD to MCI best fit a curvilinear regression line (Figure 1). Three (14\%) of these 22 subjects progressed to MCI within 3 years after baseline, and ten (63\%) of 16 subjects progressed within 7 years. The overall rate of preclinical AD progression to MCI was $10.4 \%$ per year. Two of the preclinical $\mathrm{AD}$ subjects developed $\mathrm{AD}$ dementia during the follow-up period, but none of the amyloid-negative $\mathrm{CN}$ subjects progressed to MCI or any dementia.

When preclinical AD subjects progressed to MCI, the mean MMSE score significantly decreased from $29.0 \pm 0.7$ to $25.7 \pm 2.5(\mathrm{n}=12 ; \mathrm{p}<0.05)$ and the global CDR score was 0.5 . The mean delayed paragraph recall score of converters was $4.6 \pm 3.4(\mathrm{n}=12 ; \mathrm{p}<0.05)$, which was significantly different from that of stable subjects despite their similar level of education.

Nine (56\%) of 16 preclinical AD subjects aged 65-75 years progressed to MCI. Two (66.6\%) of three preclinical $\mathrm{AD}$ subjects younger than age 64 years progressed to $\mathrm{MCI}$, whereas one $(33.3 \%)$ of three subjects older than age 76 years progressed. There was no significant difference in the progression rate of three age subgroups $\left(\chi^{2} \mathrm{p}=0.74\right)$. Five $(55 \%)$ of nine women with preclinical $\mathrm{AD}$ progressed to $\mathrm{MCI}$, which was not significantly different from seven $(53 \%)$ of 13 men (Fisher's exact $\mathrm{p}=0.63$ ). Four $(44.4 \%)$ of nine APOE $\varepsilon 4$ carriers with preclinical AD progressed to $\mathrm{MCI}$, whereas eight $(62 \%)$ of $13 \mathrm{APOE} \varepsilon 4$ noncarriers progressed. There was no significant difference in the 
Table 1. Demographic characteristics of preclinical Alzheimer's disease and amyloid-negative cognitively normal subjects at baseline and at follow-up.

\begin{tabular}{|c|c|c|c|c|}
\hline & \multicolumn{3}{|c|}{ Preclinical Alzheimer's disease } & \multirow[t]{2}{*}{ Cognitively normal } \\
\hline & All & Converter & Stable & \\
\hline $\mathrm{n}$ & 22 & 12 & 10 & 10 \\
\hline Female & $9(41 \%)$ & $5(41 \%)$ & $4(40 \%)$ & $5(50 \%)$ \\
\hline Age $(y)$ & $71.0 \pm 5.9$ & $71.1 \pm 5.8$ & $70.9 \pm 6.1$ & $65.7 \pm 3.4$ \\
\hline Education (y) & $12.1 \pm 2.6$ & $12.2 \pm 2.4$ & $11.9 \pm 2.7$ & $13.5 \pm 1.6$ \\
\hline APOE $\varepsilon 4$ carriers & $9(41 \%)$ & $4(33 \%)$ & $5(50 \%)$ & $1(10 \%)$ \\
\hline \multicolumn{5}{|l|}{ At baseline } \\
\hline MMSE & $29.1 \pm 0.8$ & $29.0 \pm 0.7$ & $29.2 \pm 0.8$ & $29.6 \pm 0.6$ \\
\hline Global CDR & 0 & 0 & 0 & 0 \\
\hline Immediate Rec & $13.3 \pm 2.5$ & $12.7 \pm 2.6$ & $14.1 \pm 2.1$ & $14.2 \pm 1.8$ \\
\hline Delayed Rec & $11.6 \pm 2.7$ & $10.6 \pm 3.0$ & $12.9 \pm 1.2$ & $12.1 \pm 3.0$ \\
\hline \multicolumn{5}{|l|}{ At the last follow-up } \\
\hline MMSE & $27.1 \pm 2.4$ & $25.7 \pm 2.5^{\dagger}$ & $28.8 \pm 0.7$ & $29.0 \pm 0.6$ \\
\hline Global CDR & $0.25 \pm 0.25$ & 0.5 & 0 & 0 \\
\hline Immediate Rec & $9.9 \pm 4.6$ & $7.1 \pm 3.8^{\dagger}$ & $13.2 \pm 3.2$ & $13.8 \pm 1.9$ \\
\hline Delayed Rec & $7.7 \pm 4.5$ & $4.6 \pm 3.4^{\dagger}$ & $11.4 \pm 2.7$ & $12.4 \pm 3.4$ \\
\hline Follow-up duration (y) & $6.0 \pm 1.8$ & $6.5 \pm 1.3$ & $5.4 \pm 2.3$ & $5.6 \pm 2.6$ \\
\hline
\end{tabular}

Data are presented as means \pm SD.

† Statistically significant difference from baseline and CN by two-sample t-tests $(p<0.05)$

APOE: Apolipoprotein E; CDR: Clinical dementia rating; CDR SB: CDR sum of boxes; MMSE: Mini-Mental State Examination; n: Number of patients; Rec: WMS-R recall scores; WMS-R: Revised Wechsler memory scale; y: Year.

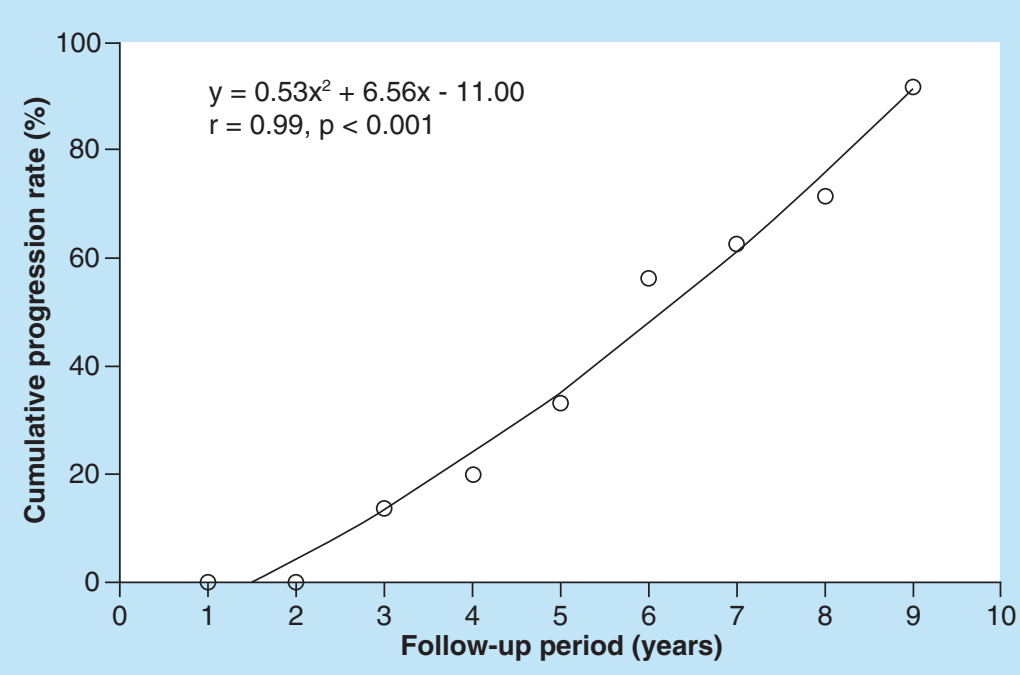

Figure 1. Curvilinear regression between the consecutive rate of progression from preclinical Alzheimer's disease to mild cognitive impairment and the follow-up period in preclinical Alzheimer's disease subjects. The linear model is $y=-11.63+7.19 x+0.46 x^{2}(r=0.99 ; p<0.001)$.

progression rate between APOE $\varepsilon 4$ carriers and noncarriers (Fisher's exact $\mathrm{p}=0.36$ ). The progression to MCI was not directly related to baseline age, gender or APOE $\varepsilon 4$ status.

\section{$\mathrm{A} \beta$ deposition}

18 of 22 preclinical AD subjects had marked $\left[{ }^{11} \mathrm{C}\right]-\mathrm{PIB}$ uptake in the FC, PC and LTC regions as well as in the cingulate gyrus and precuneus on the typical positive scan. In contrast, four subjects had focal positive scans with increased regional $\left[{ }^{11} \mathrm{C}\right]$-PIB uptake in the Pre, PC and/or FC regions. 


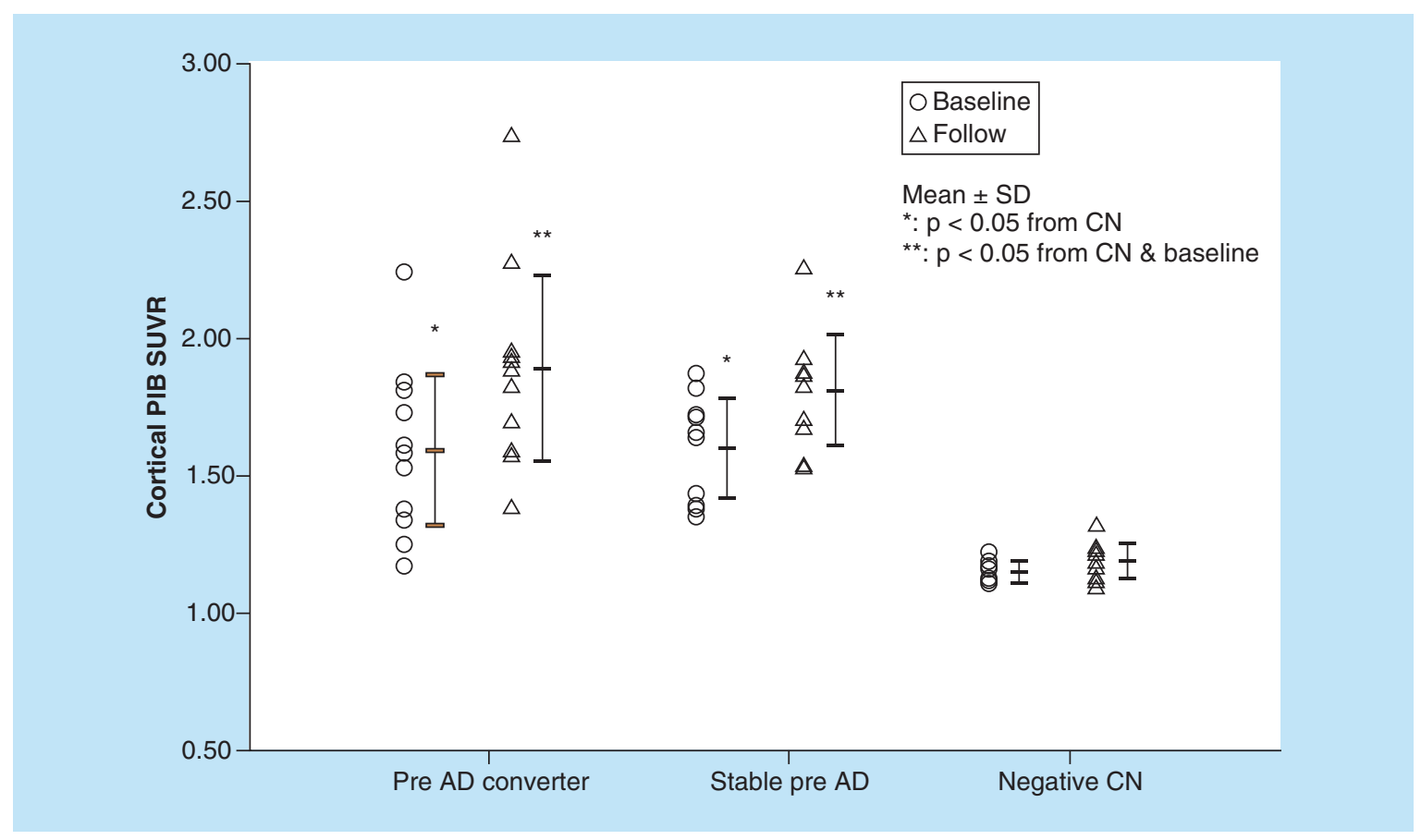

Figure 2. Cortical Pittsburgh Compound-B standardized uptake value ratio in preclinical Alzheimer's disease (pre-Alzheimer's disease) converters $(n=12)$, stable preclinical Alzheimer's disease subjects $(n=10)$ and amyloid-negative cognitively normal subjects $(n=10)$ at baseline (open circles) and follow-up (open triangles). Data are presented as the mean \pm SD.

*Statistically significant compared with CN $(p<0.05) ; * *$ Statistically significant compared with baseline and negative $\mathrm{CN}(\mathrm{p}<0.05)$.

CN: Cognitively normal; PIB: ${ }^{11}$ C-labelled Pittsburgh Compound-B; SD: Standard deviation; SUVR: Standardized uptake value ratio.

The mean value of global cortical PIB SUVRs in preclinical AD subjects at baseline $(1.60 \pm 0.23 ; \mathrm{n}=22$; $\mathrm{p}<0.01)$ was significantly higher than that in amyloid-negative $\mathrm{CN}$ subjects $(1.15 \pm 0.04 ; \mathrm{n}=10)$. The mean of the global cortical PIB SUVR at follow-up $(1.89 \pm 0.28 ; \mathrm{n}=22 ; \mathrm{p}<0.01)$ was significantly increased more than that at baseline. The annual increase in the global cortical SUVR in preclinical AD subjects over a mean follow-up of $6.0 \pm 1.8$ years was $0.054 \pm 0.020(\mathrm{n}=22 ; \mathrm{p}<0.01)$, which was significantly larger than that in amyloid-negative $\mathrm{CN}$ subjects $(0.005 \pm 0.007 ; \mathrm{n}=10)$. This change was a $3.3 \%$ annual increase in the PIB SUVR from baseline.

The global cortical PIB SUVR in APOE $\varepsilon 4$ carriers with preclinical AD at baseline $(1.59 \pm 0.28 ; n=9 ; p=0.96)$ did not significantly differ from that in APOE $\varepsilon 4$ noncarriers $(1.60 \pm 0.21 ; n=13)$. There was also no significant difference in the annual change in PIB SUVR between APOE $\varepsilon 4$ carries and noncarriers. In addition, the baseline age of individual preclinical AD subjects was not significantly related to the baseline PIB SUVR $(r=-0.23 ; n=22$; $\mathrm{p}=0.29)$ or the annual change in the PIB SUVR $(\mathrm{r}=0.14 ; \mathrm{n}=22 ; \mathrm{p}=0.52)$

When preclinical $\mathrm{AD}$ subjects were separated into two subgroups of converters and stable subjects, the mean cortical PIB SUVR at baseline in preclinical AD converters $(1.59 \pm 0.27 ; \mathrm{n}=12 ; \mathrm{p}=0.99)$ was similar to that in stable subjects $(1.60 \pm 0.18 ; \mathrm{n}=10$; Figure 2$)$. The annual increase in the global cortical SUVR in preclinical AD converters was $0.053 \pm 0.020(\mathrm{n}=12 ; \mathrm{p}=0.91)$, which was similar to $0.054 \pm 0.021(\mathrm{n}=10)$ in stable subjects (Figure 3). In contrast, the period of progression to $\mathrm{MCI}$ in individual preclinical $\mathrm{AD}$ converters was significantly related to the cortical PIB SUVR at baseline $(\mathrm{r}=-0.57 ; \mathrm{n}=12 ; \mathrm{p}<0.05)$ but not the annual increase in the cortical PIB SUVR $(\mathrm{r}=-0.52 ; \mathrm{n}=12 ; \mathrm{p}=0.08)$.

Among the cortical regions of preclinical AD subjects, the regional PIB SUVRs at baseline and the annual increases in the regional cortical PIB SUVR in all cortical regions except MTC were significantly higher than those in the amyloid-negative $\mathrm{CN}$ subjects (Table 2). The regional PIB SUVRs at baseline $(1.89 \pm 0.39 ; \mathrm{n}=22)$ and the annual increase in the regional PIB SUVR $(0.080 \pm 0.036 ; n=22)$ were greatest in the Pre. There was no 


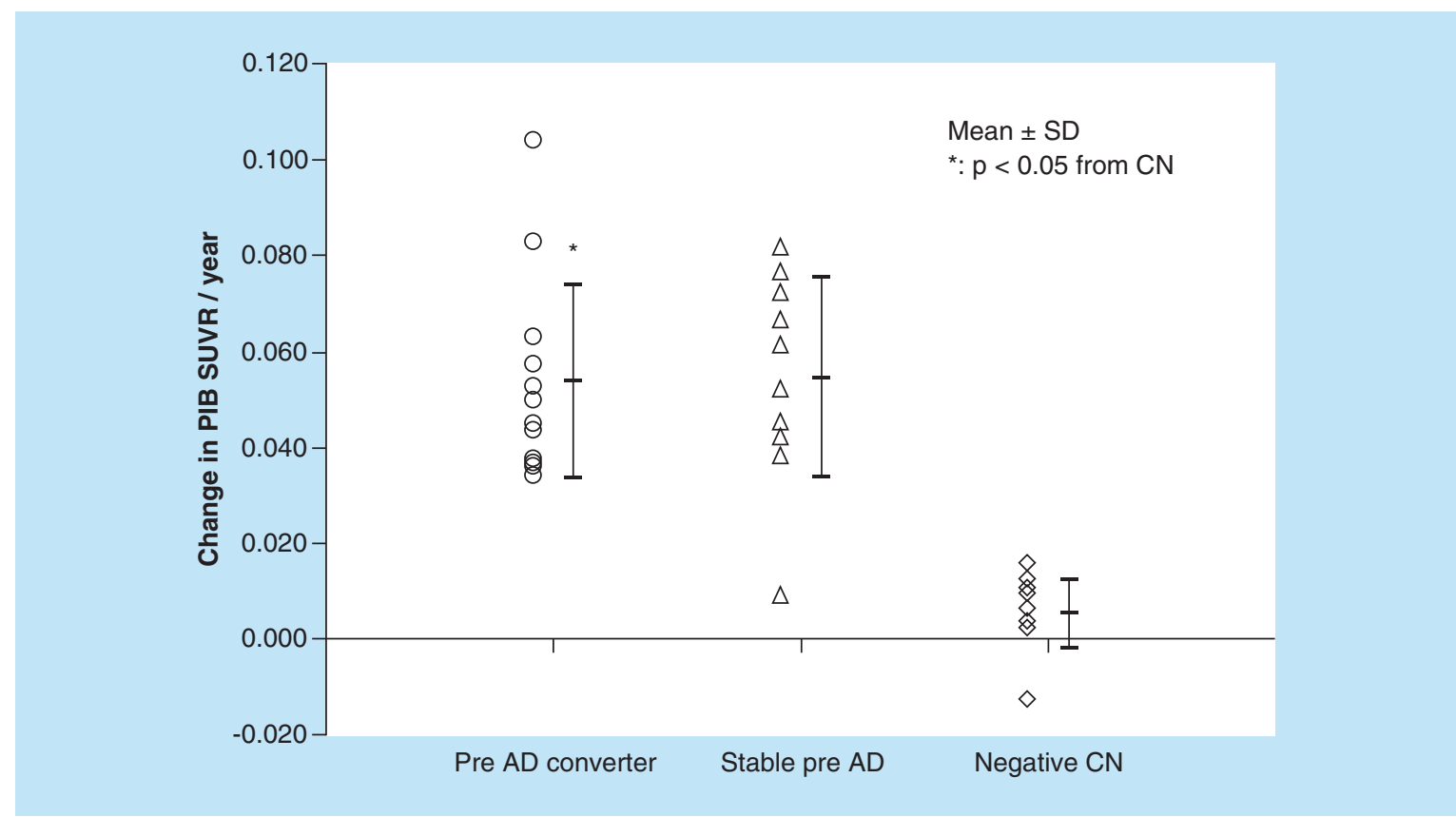

Figure 3. Annual changes in the cortical Pittsburgh Compound-B standardized uptake value ratio of preclinical Alzheimer's disease (pre-Alzheimer's disease) converters $(n=12)$, stable preclinical Alzheimer's disease subjects $(n=10)$ and amyloid-negative cognitively normal subjects $(n=10)$. The annual increase in the cortical PIB SUVR in preclinical $A D$ converters and stable subjects is significantly greater than in negative CN subjects. Data are presented as the mean \pm SD.

*Statistically significant compared with negative $\mathrm{CN}(\mathrm{p}<0.05)$.

AD: Alzheimer's disease; CN: Cognitively normal; PIB SUVR: Pittsburgh Compound-B standardized uptake value ratio; SD: Standard deviation.

Table 2. Regional Pittsburgh Compound-B standardized uptake value ratio at baseline and the annual change of regional Pittsburgh Compound-B standardized uptake value ratio in preclinical Alzheimer's disease subjects and negative-amyloid cognitively normal subjects.

\begin{tabular}{|c|c|c|c|c|}
\hline & \multicolumn{2}{|c|}{ Baseline } & \multicolumn{2}{|c|}{ Annual change } \\
\hline & Pre-Alzheimer's disease & Cognitively normal & Pre-Alzheimer's disease & Cognitively normal \\
\hline$n$ & 22 & 10 & 22 & 10 \\
\hline Medial temporal cortex & $1.18 \pm 0.12$ & $1.06 \pm 0.12$ & $0.005 \pm 0.027$ & $-0.001 \pm 0.022$ \\
\hline Lateral temporal cortex & $1.60 \pm 0.27^{\dagger}$ & $1.16 \pm 0.10$ & $0.063 \pm 0.029^{\dagger}$ & $0.004 \pm 0.012$ \\
\hline Anterior cingulate gyrus & $1.76 \pm 0.42^{\dagger}$ & $1.19 \pm 0.10$ & $0.068 \pm 0.041^{\dagger}$ & $-0.002 \pm 0.023$ \\
\hline Frontal cortex & $1.78 \pm 0.36^{\dagger}$ & $1.16 \pm 0.11$ & $0.051 \pm 0.032^{\dagger}$ & $0.006 \pm 0.0184$ \\
\hline Occipital cortex & $1.37 \pm 0.18^{\dagger}$ & $1.21 \pm 0.08$ & $0.047 \pm 0.033^{\dagger}$ & $-0.009 \pm 0.009$ \\
\hline Posterior cingulate gyrus & $1.71 \pm 0.35^{\dagger}$ & $1.22 \pm 0.12$ & $0.063 \pm 0.035^{\dagger}$ & $0.011 \pm 0.015$ \\
\hline Precuneus & $1.89 \pm 0.39^{\dagger}$ & $1.15 \pm 0.07$ & $0.080 \pm 0.036^{\dagger}$ & $0.011 \pm 0.0162$ \\
\hline Parietal cortex & $1.70 \pm 0.36^{\dagger}$ & $1.09 \pm 0.11$ & $0.060 \pm 0.038^{\dagger}$ & $0.002 \pm 0.017$ \\
\hline Sensory motor cortex & $1.45 \pm 0.28^{\dagger}$ & $1.17 \pm 0.10$ & $0.028 \pm 0.043^{\dagger}$ & $-0.002 \pm 0.015$ \\
\hline
\end{tabular}

significant difference in the regional baseline PIB SUVR and the annual change in the PIB SUVR of any cortical region between converters and stable subjects. 


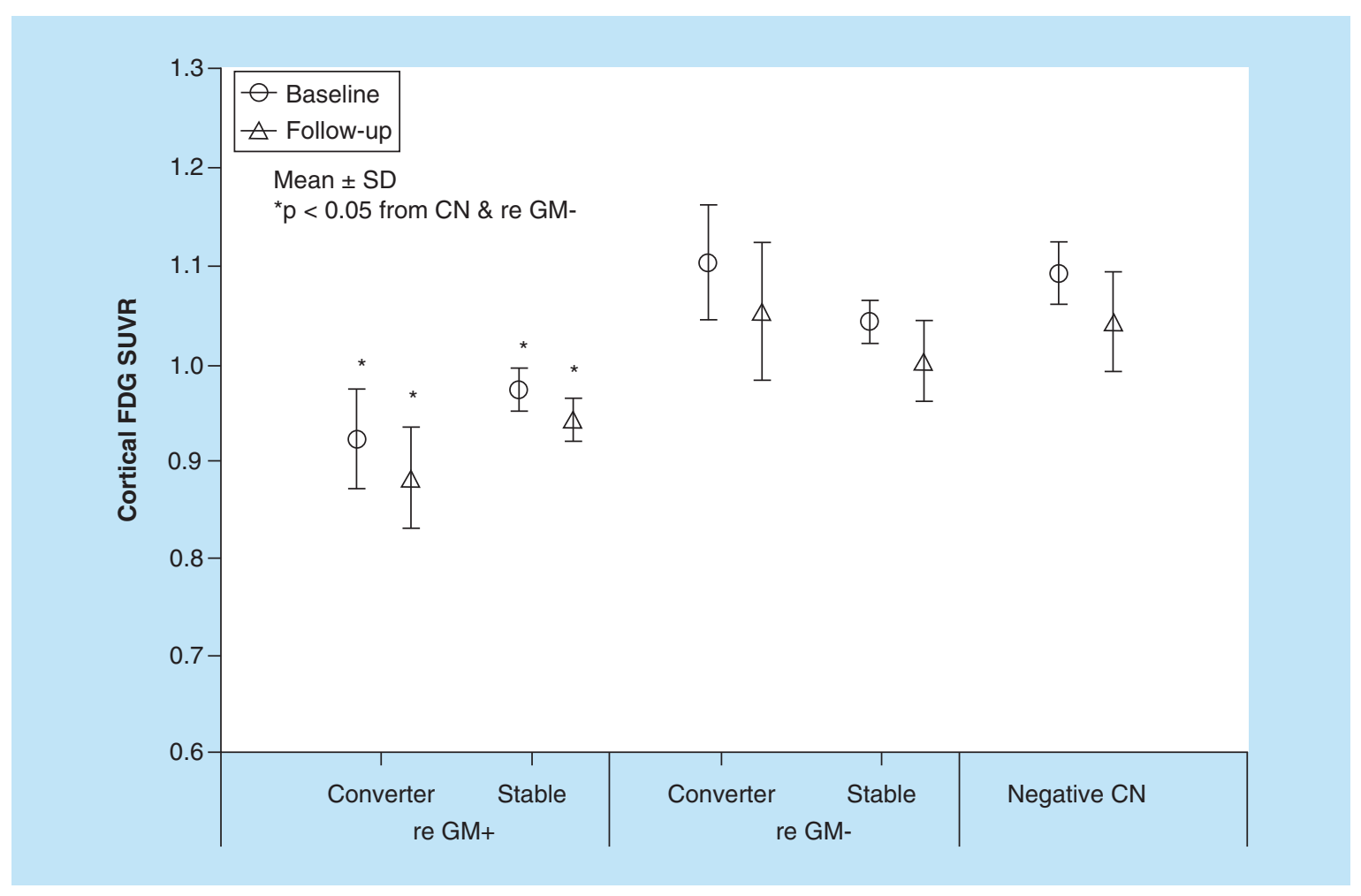

Figure 4. Cortical fluorodeoxyglucose standardized uptake value ratio in preclinical Alzheimer's disease converters and stable subjects with reduced glucose metabolism $\left(\right.$ re-GM+ $\left.{ }^{+} ; n=11\right)$ and without reduced glucose metabolism (re-GM- $n=11)$, and amyloid-negative cognitively normal subjects $(n=10)$ at baseline (open circles) and follow-up (open triangles). Data are presented as the mean \pm SD.

*Statistically significant difference with multiple comparisons post hoc tests $(p<0.05)$.

\section{Glucose metabolism}

None of the ten $\mathrm{CN}$ subjects with negative-amyloid scans had reduced glucose metabolism in any cortical region over the follow-up period. The mean global cortical FDG SUVR at baseline and follow-up were $1.07 \pm 0.02$ $(\mathrm{n}=10)$ and $1.05 \pm 0.04(\mathrm{n}=10)$, respectively (Figure 4).

$11(50 \%)$ of 22 subjects with preclinical AD had reduced glucose metabolism in AD-affected regions, such as the LTC, PC and/or Pre. The mean global cortical FDG SUVR decreased from $0.94 \pm 0.05$ at baseline to $0.91 \pm 0.05(\mathrm{n}=11, \mathrm{p}<0.01)$ at follow-up (Figure 4). The mean annual change in the cortical FDG SUVR was $-0.006 \pm 0.006(n=11)$. In contrast, the 11 subjects without reduced glucose metabolism had a global FDG SUVR of $1.07 \pm 0.05$ at baseline, which did not change at follow-up. Six (54\%) of 11 preclinical AD subjects with reduced glucose metabolism progressed to MCI, which was not significantly different from the six (54\%) of 11 subjects without reduced glucose metabolism (Fisher's exact $\mathrm{p}=0.66$ ). In preclinical AD subjects with reduced glucose metabolism, there was no significant difference in the baseline FDG SUVR or the annual change in FDG SUVR between converters and stable subjects.

Of the AD-affected cortical regions, seven (58\%) of 12 subjects with reduced glucose metabolism (FDG SUVR: $0.94 \pm 0.04 ; \mathrm{n}=12)$ in the LTC progressed to MCI, whereas five (50\%) of ten subjects without reduced glucose metabolism progressed. In addition, three (43\%) of seven subjects with reduced glucose metabolism (FDG SUVR: $0.93 \pm 0.04 ; \mathrm{n}=7)$ in the PC progressed to $\mathrm{MCI}$, whereas nine (60\%) of 15 subjects without reduced glucose metabolism progressed. There was no significant difference in the rate of $\mathrm{MCI}$ progression between subjects with and without reduced glucose metabolism in the LTC (Fisher's exact $\mathrm{p}=0.51$ ) or in the PC (Fisher's exact $\mathrm{p}=0.38$ ). The reduced glucose metabolism in $\mathrm{AD}$-affected regions of preclinical $\mathrm{AD}$ subjects was not related to the progression to MCI. 


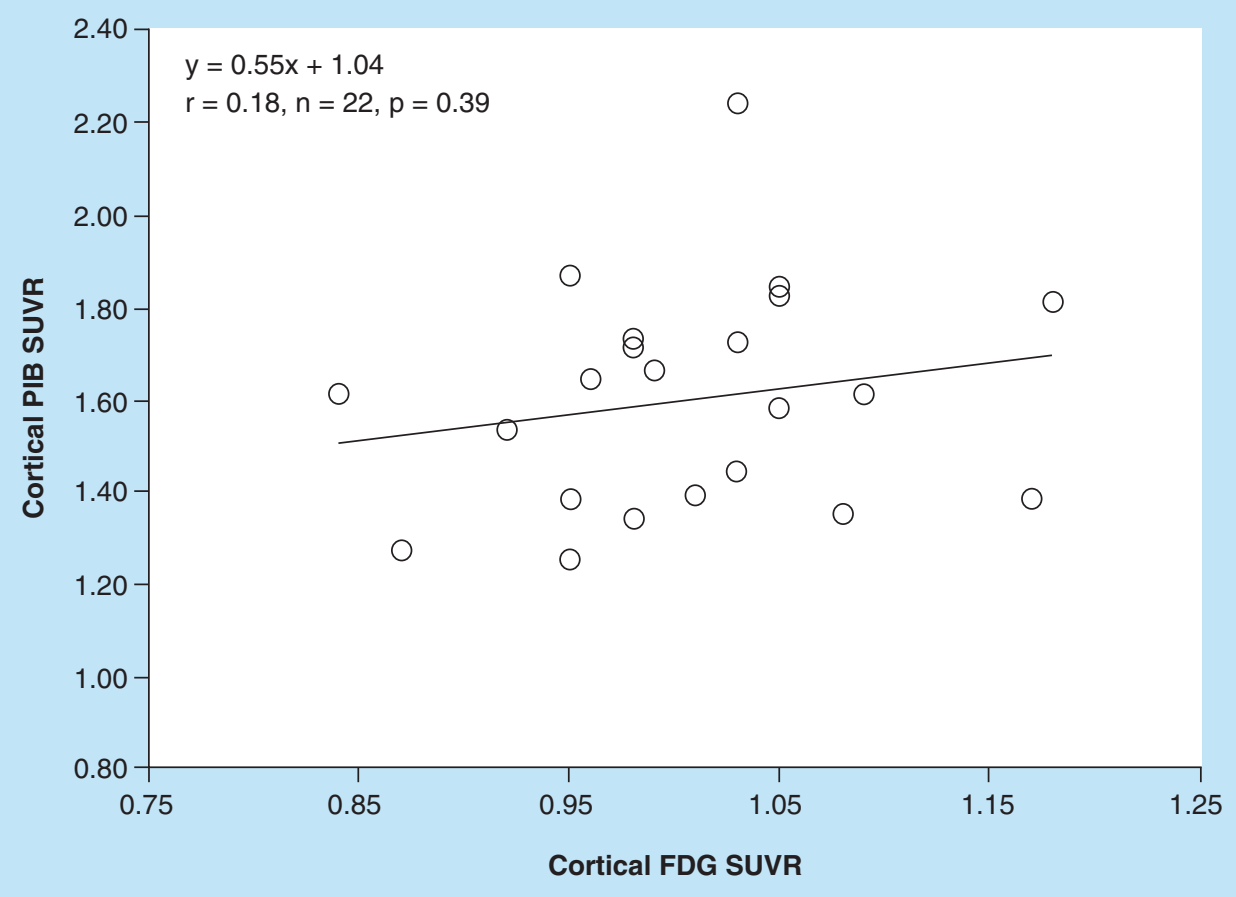

Figure 5. Relationship between cortical Pittsburgh Compound-B and fluorodeoxyglucose standardized uptake value ratio values at baseline in individual preclinical Alzheimer's disease subjects. Cortical PIB SUVRs are not significantly correlated with cortical FDG SUVRs $(r=0.18, n=22 ; p=0.39)$.

FDG SUVR: Fluorodeoxyglucose standardized uptake value ratio; PIB SUVR: Pittsburgh Compound-B standardized uptake value ratio.

\section{A $\beta$ deposition \& glucose metabolism}

The relationship between the global cortical PIB SUVR and the FDG SUVR in individual preclinical AD subjects at baseline is shown in Figure 5. Individual cortical PIB SUVRs were not significantly correlated with cortical FDG SUVRs in preclinical AD subjects $(\mathrm{r}=0.21 ; \mathrm{n}=22 ; \mathrm{p}=0.33)$. Annual changes in the cortical PIB SUVR in individual preclinical AD subjects were not significantly correlated with those in the cortical FDG SUVR $(\mathrm{r}=$ $-0.04 ; \mathrm{n}=22 ; \mathrm{p}=0.85$; Figure 6). Among AD-affected cortical regions, there were no significant differences in the baseline value or the annual change between the PIB SUVR and the FDG SUVR in the PC or LTC.

In preclinical AD converters, the individual cortical PIB SUVRs at baseline were not significantly correlated with cortical FDG SUVRs $(\mathrm{r}=0.32 ; \mathrm{n}=12 ; \mathrm{p}=0.30)$. In addition, the individual annual change in the cortical PIB SUVR was not correlated to that in the cortical FDG SUVR $(r=0.04 ; \mathrm{n}=12 ; \mathrm{p}=0.96)$.

\section{Discussion}

We demonstrated that 12 (55\%) of 22 subjects with preclinical AD progressed to MCI during $6.0 \pm 1.8$ years of long-term follow-up. The age, gender and APOE $\varepsilon 4$ status of the subjects were not associated with the progression to MCI. A previous study described that the rate of progression to MCI was $18 \%$ over a short follow-up of 15 months in 90 cognitively normal participants with amyloid positivity (preclinical AD stages 1-3) [8]. Additionally, the Australian Imaging, Biomarkers and Lifestyle research group reported that eight (25\%) of 32 healthy individuals with positive $A \beta$ PET scans progressed to MCI by 3 years [12]. Our findings indicate that a higher proportion of the $\mathrm{CN}$ subjects with $\mathrm{A} \beta$ deposition have progression to $\mathrm{MCI}$ if we have much longer observation periods. It is suggested that individuals with preclinical $\mathrm{AD}$ progress to $\mathrm{MCI}$ over the long term when preclinical $\mathrm{AD}$ is defined by positive PET imaging of $A \beta$ biomarkers, although some older individuals with preclinical $A D$ may not become symptomatic during their lifetime. Furthermore, the present study showed that the overall rate of preclinical AD progression to $\mathrm{MCI}$ was $10.4 \%$ per year. This rate is lower than the $23.4 \%$ per year of $\mathrm{MCI}$ due to $\mathrm{AD}$ progression 


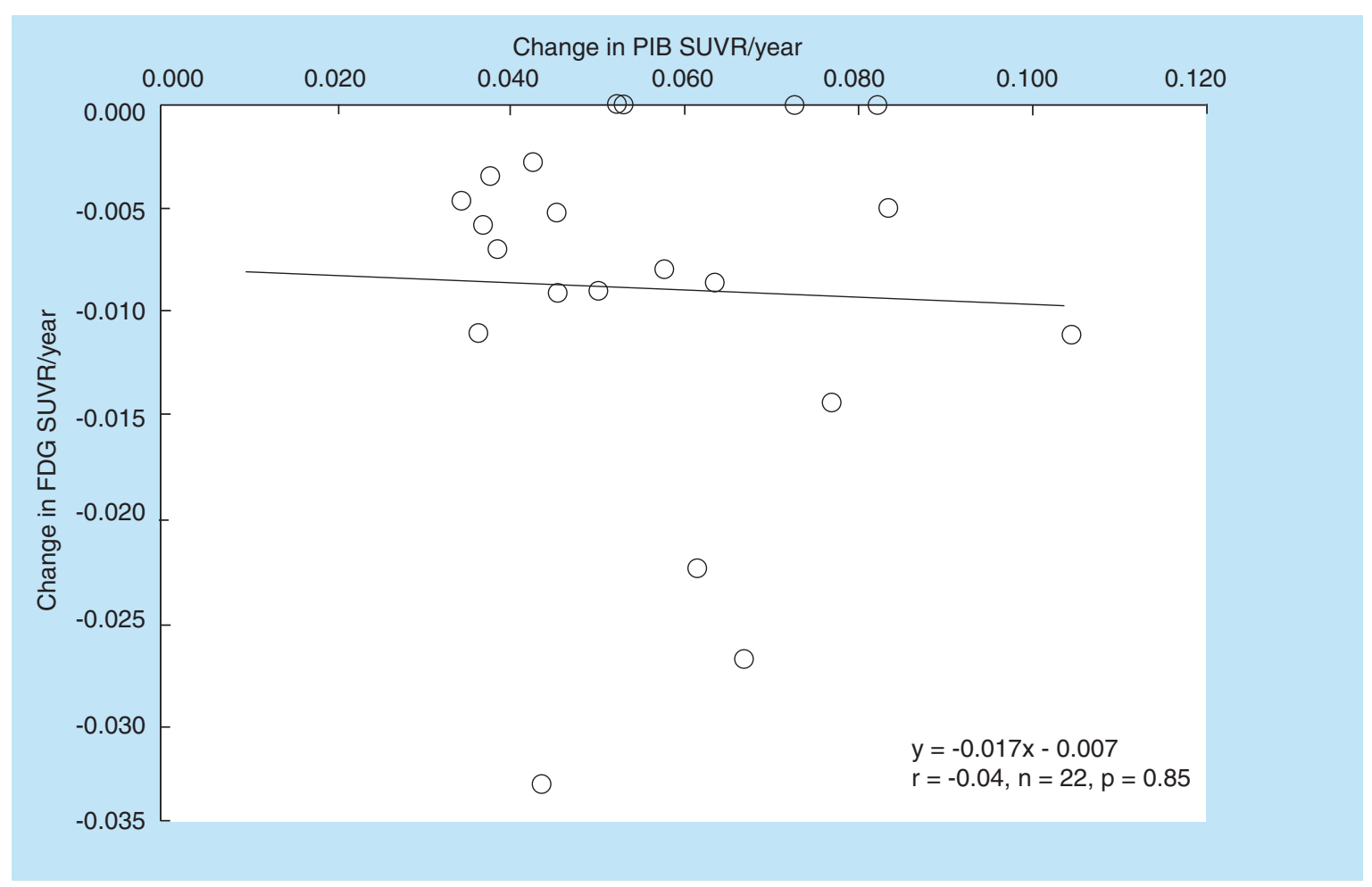

Figure 6. Relationship between annual changes in the cortical Pittsburgh Compound-B standardized uptake value ratio and fluorodeoxyglucose standardized uptake value ratio in individual preclinical Alzheimer's disease subjects. The annual change in the PIB SUVR is not significantly correlated with that in the FDG SUVR $(r=-0.04 ; n=22 ; p=0.85)$. FDG SUVR: Fluorodeoxyglucose standardized uptake value ratio; PIB SUVR: Pittsburgh Compound-B standardized uptake value ratio.

to $\mathrm{AD}$ that was previously reported [5]. Subjects with preclinical $\mathrm{AD}$ cloud progress more slowly than subjects with $\mathrm{MCI}$ due to $\mathrm{AD}$.

When subjects with preclinical AD had reduced glucose metabolism with FDG PET imaging at baseline, the present study found that six (54\%) of 11 subjects with reduced glucose metabolism progressed to MCI over a long follow-up of $6.0 \pm 1.8$ years. This rate of progression did not significantly differ from the six $(54 \%)$ of 11 subjects without reduced glucose metabolism. A previous study reported that the rate of conversion to $\mathrm{MCI}$ in 25 individuals with preclinical AD stages $2+3$ with $\beta$-amyloidosis and lower glucose metabolism was $21 \%$ over a short follow-up of 15 months, which was not different from the $19 \%$ of 32 individuals with preclinical AD stage 1 with $\beta$-amyloidosis alone [13]. We suggest, in subjects with preclinical $\mathrm{AD}$, that a reduction in cerebral glucose metabolism at baseline could not be predict of a progression to $\mathrm{MCI}$ even over a longer time.

We demonstrated that the global cortical PIB SUVR in preclinical AD subjects at follow-up significantly increased from baseline. The annual increase in the PIB SUVR was $0.054 \pm 0.020$ and $3.3 \%$ from baseline. This is consistent with a longitudinal PIB PET study that PIB SUVR was $0.046 \pm 0.03$ per year in healthy controls with PIB retention [14]. These findings indicate that $A \beta$ deposition continues to accumulate over time in subjects with preclinical AD. However, the present study found that the annual increase in $A \beta$ deposition in the preclinical AD converters did not significantly differ from that that in stable subjects during the follow-up period. On the other hand, the cortical PIB SUVR at baseline in preclinical AD converters was correlated with the period of progression to $\mathrm{MCI}$. These findings suggest that subjects with preclinical $\mathrm{AD}$ can progress to $\mathrm{MCI}$ even over a shorter period when they have higher $A \beta$ deposition at baseline in addition to a continuous increase in $A \beta$ deposition.

We found that $11(50 \%)$ of 22 preclinical $\mathrm{AD}$ subjects had reduced cerebral glucose metabolism at baseline in AD-affected regions of the LTC, PC and/or Pre. The mean cortical FDG SUVR significantly decreased from $0.94 \pm 0.05$ at baseline to $0.91 \pm 0.05$ during a long follow-up of 6 years, and the annual change in the FDG SUVR was $-0.0061 \pm 0.0060$. This result is consistent with a previous study that the hypometabolism in the AD signature composite regions at baseline decreased over a short follow-up of 15 months in $43 \%$ of CN participants 
with amyloid deposition [15]. In addition, the present study showed, in preclinical AD subjects with reduced glucose metabolism, that there was no significant difference in the baseline FDG SUVR or the annual change in the FDG SUVR between converters and stable subjects. These findings indicate that a change in glucose metabolism is not associated with the progression to MCI. We suggest that if preclinical AD subjects have reduced glucose metabolism in $\mathrm{AD}$-affected regions at baseline the glucose metabolism could further decrease over time without being directly involved in the progression to MCI.

The present study demonstrated that the cortical PIB SUVRs at baseline were not significantly correlated with cortical FDG SUVRs in individual preclinical AD subjects. Furthermore, the annual increase in A $\beta$ deposition was not significantly related to the annual decrease in glucose metabolism. These findings support the previous study that the abnormal levels of $\mathrm{A} \beta$ did not result in greater hypometabolism in $\mathrm{CN}$ individuals [6]. In $\mathrm{AD}$ patients, in contrast, higher $A \beta$ retention has already established to be correlated with lower glucose metabolism in the temporal and parietal cortices. Early in the preclinical stage of $\mathrm{AD}$ spectrum, the volume and period of increased $\mathrm{A} \beta$ deposition may be not sufficient to directly promote hypometabolism. It appears that there are different upstream causes for $\mathrm{A} \beta$ production and glucose metabolism.

We demonstrated that subjects with preclinical $\mathrm{AD}$ more frequently progressed to $\mathrm{MCI}$ over a long follow-up period when preclinical $A D$ was defined by a positive PIB PET scan of the A $\beta$ biomarker. In addition, our study showed that $A \beta$ deposition continuously increased over time in preclinical $A D$ subjects, but the increase in $A \beta$ deposition was not directly related to decrease in glucose metabolism even in AD-affected cortical regions. The $\mathrm{A} \beta$ deposition may be the primary event that induces other neurodegenerative processes, such as the accumulation and/or spreading of Tau, in the early stage of preclinical AD. Therefore, if preclinical AD is defined by amyloid PET imaging, a great success may be achieved with anti-amyloid agents by decreasing the production and accumulation of $A \beta$ very early in the disease process, before clinical impairment.

Certain limitations of our study should be noted. We conducted a successful longitudinal assessment of $A \beta$ deposition and glucose metabolism on the progression of preclinical $\mathrm{AD}$ subjects during a long-term follow-up period, but the number of preclinical AD subjects was small. The study population may have been limited by the definition of $\mathrm{CN}$ and the threshold for defining amyloid positivity and reduced glucose metabolism in our clinical setting.

\section{Conclusion}

Preclinical AD subjects have continuous increases in $A \beta$ deposition over time independent of glucose metabolism. Increased $A \beta$ deposition leads to a progression to MCI but reduced glucose metabolism does not contribute to the progression. Subjects with preclinical AD defined by amyloid PET are at a higher risk for progression to MCI or $\mathrm{AD}$ dementia over the long term.

\section{Future perspective}

Cognitively normal subjects progress to $\mathrm{MCI}$ or $\mathrm{AD}$ over a long period if they have $\mathrm{A} \beta$ deposition as evaluated by a positive amyloid PET scan. The A $\beta$ deposition continuously increases over time and induces other neurodegenerative processes. Antiamyloid agents that decrease the production and/or accumulation of $A \beta$ should be developed in the future, and are needed very early in the disease process, before clinical impairment.

\section{Summary points}

- Subjects with preclinical Alzheimer's disease (AD) progress to mild cognitive impairment (MCl) over the long term if preclinical $A D$ is defined by amyloid positron emission tomography imaging.

- The cortical Pittsburgh Compound-B standardized uptake value ratio (PIB SUVR) is higher at baseline and increases during follow-up period in preclinical AD.

- The annual increase in the PIB SUVR is 3.3\% from baseline.

- In preclinical AD subjects who progressed to $\mathrm{MCl}$, the baseline value or the annual change in cortical PIB SUVR is not significantly correlated with that in cortical fluorodeoxyglucose SUVR.

- A reduction in cerebral glucose metabolism is not correlated with a progression to $\mathrm{MCl}$ even over a longer time.

- Increases in amyloid $\beta$ deposition lead to the progression to $\mathrm{MCl}$ but decreases in glucose metabolism do not contribute to progression in the preclinical stage of $A D$ spectrum. 


\section{Acknowledgments}

The authors gratefully acknowledge all individuals who participated in this study.

\section{Financial \& competing interests disclosure}

The authors have no relevant affiliations or financial involvement with any organization or entity with a financial interest in or financial conflict with the subject matter or materials discussed in the manuscript. This includes employment, consultancies, honoraria, stock ownership or options, expert testimony, grants or patents received or pending, or royalties.

No writing assistance was utilized in the production of this manuscript.

\section{Ethical conduct of research}

The authors state that they have obtained appropriate institutional review board approval or have followed the principles outlined in the Declaration of Helsinki for all human or animal experimental investigations. In addition, for investigations involving human subjects, informed consent has been obtained from the participants involved. The authors state that they have obtained verbal and written informed consent from the patient/patients for the inclusion of their medical and treatment history within this case report.

\section{Open access}

This work is licensed under the Creative Commons Attribution 4.0 License. To view a copy of this license, visit http://creativecomm ons.org/licenses/by/4.0/

\section{References}

1. Jack CR, Albert MS, Knopman DS et al. Introduction to the recommendations from the National Institute on Aging-Alzheimer's Association Workgroups on diagnostic guidelines for Alzheimer's disease. Alzheimers Dement. 7, 257-262 (2011).

2. Sperling RA, Aisen PS, Beckett LA et al. Toward defining the preclinical stages of Alzheimer's disease: Recommendation from the National Institute on Aging-Alzheimer's Association workgroups on diagnostic guidelines for Alzheimer's disease. Alzheimers Dement. 7 , 280-292 (2011).

3. Klunk WE, Engler H, Nordberg A et al. Imaging brain amyloid in Alzheimer's disease with Pittsburgh Compound-B. Ann. Neurol. 55 , 306-319 (2004).

4. Hatashita S, Yamasaki H. Clinically different stages of Alzheimer's disease associated by amyloid deposition with [11C]-PIB PET imaging. J. Alzheimers Dis. 21, 995-1003 (2010).

5. Hatashita $S$, Wakebe $D$. Amyloid- $\beta$ deposition and long-term progression in mild cognitive impairment due to Alzheimer's disease defined with amyloid PET imaging. J. Alzheimers Dis. 57, 765-773 (2017).

6. Lowe VJ, Weigand SD, Senjem ML et al. Association of hypometabolism and amyloid levels in aging, normal subjects. Neurology 82 , 1959-1967 (2014).

7. Hatashita S, Yamasaki H. Diagnosed mild cognitive impairment due to Alzheimer's disease with PET biomarkers of beta amyloid and neuronal dysfunction. PLoS ONE 14, e66877 (2013).

8. Knopman DS, Jack CR Jr, Wiste HJ et al. Short-term clinical outcomes for stages of NIA-AA preclinical Alzheimer disease. Neurology 78, 1576-1582 (2012).

9. Rowe CC, Bourgeat P, Ells KA et al. Predicting Alzheimer disease with $\beta$-amyloid imaging: results from the Australian imaging, biomarkers, and lifestyle study of ageing. Ann. Neurol. 74, 905-913 (2013).

10. Albert MS, DeKosky ST, Diskson D et al. The diagnosis of mild cognitive impairment due to Alzheimer's disease: recommendation from the National Institute on Aging-Alzheimer's Association workgroups on diagnostic guidelines for Alzheimer's disease. Alzheimers Dement. 7, 270-279 (2011).

11. Hatashita S, Yamasaki H, Suzuki Y et al. [18F] Flutemetamol amyloid-beta PET imaging compared with $\left[{ }^{11} \mathrm{C}\right]$ PIB across the spectrum of Alzheimer's disease. Eur. J. Nucl. Med. Mol. Imaging 41, 290-300 (2014).

12. Villemagne VL, Pike KE, Chetelat G et al. Longitudinal assessment of $\mathrm{A} \beta$ and cognition in aging and Alzheimer disease. Ann. Neurol. 69, 181-192 (2011).

13. Knopman DS, Jack CR Jr, Wiste HJ et al. Selective worsening of brain injury biomarker abnormalities in cognitively normal elderly with $\beta$-amyloidosis. JAMA Neurol. 70, 1030-1038 (2013).

14. Jack Jr CR, Wiste HJ, Lesnick TG et al. Brain B-amyloid load approaches a plateau. Neurology 80, 890-896 (2013).

15. Villemagne VL, Bumham S, Bourgeat $\mathrm{P}$ et al. Amyloid $\beta$ deposition, neurodegeneration, and cognitive decline in sporadic Alzheimer's disease: a prospective cohort study. Lancet Neurol. 12, 357-367 (2013). 
